\title{
El periodismo educativo: objetivos
}

\author{
Dr. Guillermo Raigón Pérez de la Concha \\ Profesor de Periodismo Educativo \\ Universidad de Sevilla
}

Además de una especialidad sectorial o temática de la investigación y de la práctica profesional, el Periodismo Educativo es una reflexión sistemática sobre los aspectos educadores de la información de actualidad, coadyuvante necesario de una orientación social contemporánea y determinante que llamamos Sociedad Educativa o Educación para Toda la Vida. En el artículo se describen los objetivos fundamentales de dicho Periodismo, se distinguen de los característicos de la Educomunicación, se establece su temario y se propone una selección bibliográfica (1990-1998), en la que se segrega lo periodístico de lo pedagógico y didáctico.

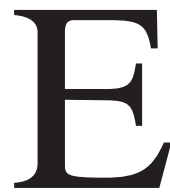

1 Periodismo Educativo es, antes que nada, producción y distribución de mensajes informativos de actualidad que logren interesar a un público determinado. El contenido de tales mensajes es la vida humana haciéndose en todas partes y bajo todas las circunstancias. Este ir haciéndose es, seguramente, la perspectiva sobre la educación como proceso continuo e inacabable que mejor cuadra a nuestra disciplina. Los hombres nos vamos haciendo cuando desarrollamos todas nuestras facultades, cuando orientamos nuestros sentimientos de dolor y de placer hacia el orden ético o hacia la mejor felicidad o hacia una vida para los demás, cuando transmitimos los modelos por los que el mundo pueda ser explicable o cuando nos vamos adaptando a ellos ${ }^{1}$.

\footnotetext{
Son lecturas esenciales para estas concepciones de la educación las siguientes: ROUSSEAU, El Emilio, Alianza, Madrid, 1990; PESTALOZZI, Cartas sobre educación infantil, Tecnos, Madrid, 1996; ROGERS, El proceso de convertirse en persona, Paidós, Buenos Aires, 1972; FRÖBEL, La educación del hombre, Daniel Jorro, Madrid, 1913; NEILL, Summerhill: un punto de vista radical sobre la educación e los niños, FCE, México, 1974; FREIRE, Pedagogía del oprimido, Siglo XXI, Madrid, 1995; DEWEY, Democracia y educación, Morata, Madrid, 1995; DURKHEIM, Educación y sociedad, Península, Barcelona, 1989.
} 
El periodista hace Periodismo Educativo, por tanto, cuando contempla la actualidad teniendo en cuenta el beneficio de los públicos: hacerles descubrir o reforzar sus posibilidades personales de participar responsablemente en la vida social, de amar y de ser amados tanto como de ser felices. Cuando informa, puede contribuir a «formar un hombre capaz de elevarse al nivel de la civilización moderna, o sea, de encontrar el sentido de la vida en este nuevo mundo» ${ }^{2}$.

Esta perspectiva de la educación a disposición de los periodista es complementaria de la que se limita a aplicar procedimientos sistemáticos de enseñanza-aprendizaje, incluida lo que la UNESCO ha llamado «educación para toda la vida» ${ }^{3}$, horizonte más ancho, incluso, que el de la educación permanente ${ }^{4}$.

La Educación para Toda la Vida coincide con la noción de sociedad educativa, «en la que -según la UNESCO- todo puede ser ocasión para aprender y desarrollar las capacidades del individuo tanto si se trata de brindar una segunda o una tercera ocasión educativa o la de satisfacer la sed de conocimientos, de belleza o de superación personal como de perfeccionar y ampliar los tipos de formación estrictamente vinculados con las exigencias de la vida profesional, comprendidos los de formación práctica». La idea de esta sociedad educativa empieza a concebirse cuando las tradiciones van perdiendo su valor de puntos de referencia colectivos y no queda más remedio que anclarse en una cierta configuración de los conocimientos adquiridos para «orientarse, pensar y actuar».

Lo cual invita a relevar la función social de los periodistas como facilitadores y promotores de conocimientos y de relatos de actualidad que puedan ser incorporados a procesos de sentido, racionales y personales o no.

Si ahora describimos algunas de las características de la Educación para Toda la Vida, se aclarará aún más nuestra concepción del Periodismo Educativo.

2 SUCHOLDOSKI, La educación humana del hombre, Laia, Barcelona, 1977. Hay que profundizar en lo que MARTÍNEZ ALBERTOS, en La noticia y los comunicadores públicos, llamaba su «preocupación básica»: que sus mensajes tengan algún grado de utilidad para los públicos a quienes van dirigidos. La que se propone el mejor Periodismo de Servicio y una de las finalidades del contenido de las noticias: «configurar públicos activos, sociedades articuladas, opiniones vertebradas en base a conocimientos nuevos de la actualidad» (Cfr. PONCE RUIZ, M., Teoría y crítica de la noticia, Padilla, Sevilla, 1995).

UNESCO, La educación encierra un tesoro, Santillana, Madrid, 1998.

4 GARCÍA ARETIO define el concepto de Educación Permanente como «el principio organizador de la educación, que pretende hacerla llegar, en todos sus niveles y modalidades, a toda la población, a lo largo de la vida, con la colaboración de los diversos sectores, instituciones y agentes» (La educación. Teoría y conceptos. Perspectivas integradoras, Paraninfo, Madrid, 1989). 
En primer lugar, es integradora porque incluye modelos de educación formal, no formal e informal, que se inspiran y corrigen entre sí. La primera es, en resumidas cuentas, la escolar (profesionalizada, reglada y graduada hasta la acreditación); la segunda es «el conjunto de procesos, medios e instituciones específicas y diferenciadamente diseñados en función de explícitos objetivos de formación o de instrucción, que no están directamente dirigidos a la provisión de los grados propios del sistema educativo reglado» 5 . Los contenidos de la educación informal son el conglomerado de cuanto de significativo nos rodea a todos en la vida cotidiana y cuya simple enumeración sería imposible. Es el medio humano como instigador multiforme y caótico de sentido.

El Periodismo Educativo se interesa por todas y cada una de las realizaciones de estos modelos: respectivamente, información sobre la Escuela; sobre actividades, instituciones y personajes que de modo sistemático se han propuesto finalidades didácticas; y sobre el haz de instancias - incluyentes y excluyentes a la vez- que son las familias, la calle y los medios de comunicación.

Con todo, el periodista sobre educación tiene hoy por hoy el deber de informar con solvencia y toda clase de precauciones sobre cuanto sucede en la Escuela, porque sigue siendo verdad que es ahí -y no en la calle ni en los medios de comunicación- donde las instancias señaladas deben encontrar un acuerdo. Además, el mundo escolar es de una complejidad tal que los usuarios necesitan explicaciones continuamente, si no queremos que el propio sistema educativo disuada a los públicos de la imprescindible estima que se le debe. Sin ella, carecería de utilidad e iría convirtiéndose en institución residual. Un trabajo informativo bien hecho puede librarnos o puede aplazar una desescolarización psicológica que está afectando especialmente a cuantos niños y jóvenes no puedan inscribirse en escuelas prestigiosas. Dígase lo mismo con respecto al estatus de semiprofesionalidad en el que se está posicionando al docente.

En realidad, la Educación para Toda la Vida presupone una enseñanza escolar eficaz, es decir, que en ella se aprenda a aprender, lo que significa que los estudiantes adquieran los instrumentos de la comprensión (atención, memoria y pensamiento). Si este cimiento es verdaderamente sólido, durante el resto de la vida irán aprendiendo a hacer (a influir sobre el propio

5 TRILLA, J., La educación fuera de la escuela. Ámbitos no formales y educación social, Ariel, Barcelona, 1993. 
entorno) y a vivir juntos (conocimiento del otro a partir del conocimiento de sí mismo, mecanismos de solución de conflictos, etc.) utilizando para ello los servicios de instituciones educativas no formales e informales ${ }^{6}$.

Los periodistas dedicados al tema educación tendrán que observar con atención estereofónica si efectivamente se cumple el derecho a una educación formal de calidad y los correlativos -y graves- deberes de los usuarios (aprender). Después tendrá que informar con una independencia que la demagogia generalizada la hará francamente difícil. Por eso, sus fuentes habrán de ser muy variadas y seguras tanto como profundo y detallado su conocimiento de las leyes sobre educación.

Un Periodismo Educativo concebido así no puede sumarse simplemente a otras disciplinas en los marcos académicos habituales ${ }^{7}$, porque los abarca todos sin excepción. Consiguientemente, no puede tratarse de una especialización más del Periodismo, por lo menos tal como, por ejemplo, lo entiende el profesor MUÑOZ TORRES, para quien el Periodismo Especializado «estudia la producción de mensajes informativos que divulgan las distintas especialidades del saber humano, de manera comprensible e interesante, al mayor número posible de personas, con el fin de dotar de sentido a la realidad, a través de los medios de comunicación» ${ }^{8}$.

Entendido así, el Periodismo Educativo quedaría reducido a información sobre los progresos de las Ciencias de la Educación y sobre sus -casi siempre- deslumbrantes aplicaciones e implicaciones ${ }^{9}$. Lo que no significa que no deba de cumplir esta tarea divulgadora porque el ciudadano desea que alguien le informe bien de la suerte de sus hijos en colegios e institutos y porque -también aquí- el periodista tiene la obligación de mostrar aspectos

6 Este punto de vista es distinto al de la simultaneidad en la Escuela que UNESCO y las últimas disposiciones legislativas en casi todo el mundo atribuyen a ese aprender a conocer, a hacer y a vivir juntos, aunque coincide con clamores de los docentes en ejercicio.

7 En las universidades españolas se cursan materias con temáticas como las siguientes: información, periodismo o comunicación sobre política y actividad parlamentaria y judicial, sobre economía y finanzas, sobre deportes, sobre producciones culturales y científico-técnicas, sobre salud y medio ambiente, sobre educación, sobre medios audiovisuales, sobre religión y sobre defensa y seguridad. Incluso se estudia «Periodismo Social» (Cfr. GÓMEZ Y MÉNDEZ; J.M., «La formación universitaria del periodista especializado», en ESTEVE RAMÍREZ, F. (coord.), Información periodística especializada, Fundación Universitaria San Pablo-CEU, Valencia, 1997.

8 «Aproximación al concepto de Información Periodística Especializada», en ESTEVE RAMÍREZ, op. cit.

9 Por ejemplo, en esta década de los noventa asistimos en todo el mundo a prolijas transformaciones legislativas que requieren de un conocimiento preciso y de unas perspectivas sociopolíticas y económicas que expliquen sus desarrollos y disfunciones demasiado alejadas de los no expertos. Por otro lado, los excesos de la nomenclatura pedagógica hacen de cualquier acontecimiento educacional algo inasequible para el no iniciado, que se limitará a reproducir -sin aclarar- lo que efectivamente pasa o se piensa. 
generalmente desconocidos y que, en su momento, pueden ser de utilidad a una parte del público ${ }^{10}$

Las tareas esbozadas no pueden depender de la presencia en la redacción de uno o varios periodistas con ideas muy claras sobre cómo funciona o cómo debe de funcionar la sociedad educativa. Es precisa y urgente una toma de conciencia de que esta nueva realidad exige nuevos enfoques narrativos y unos modos de presentación más adaptados a las posibilidades cognitivas de los públicos. Lo que exige un conocimiento estricto de niveles culturales para no infravalorar lo que sabe el público ni para sobrepasar los umbrales de conocimiento de los que dispone. No basta ya, por tanto, que los diseñadores de la información averigüen qué quiere saber el público sino cómo puede hacérselo saber.

¿Queremos decir con todo ello que la redacción de informativos en la sociedad educativa se asemejaría a la cátedra de un aula tan grande como el mundo? Sí, con tal de que se amplíe el significado de la palabra cátedra a una especie de unidad dinamizadora de la consciencia pública. Pero las finalidades, los ritmos, los agentes y los medios de los procesos informativo-periodísticos y de los pedagógicos son diferentes, aunque llamados al diálogo en bien de los públicos.

La Prensa no puede mostrar el mundo más que como va revelándose a través de acontecimientos (la actualidad); no expone sus resultados más que cada cierto tiempo (periodicidad); no es una escuela de papel y ondas o cables; y los periodistas no son profesores, aunque los mejores enseñan muy bien.

La Escuela, por su parte, no puede polarizarse en la actualidad y en el pensamiento común; su actividad no es el resultado de un proyecto empresarial y mercantil; los conocimientos que expone forman parte de un currículum complejo que procura adaptarse a lo que los docentes saben y a lo que los alumnos pueden soportar.

\footnotetext{
${ }^{10}$ Por ejemplo, las posibilidades extremas de individuación que empiezan a ofrecer las administraciones educacionales (programaciones adaptadas a la realidad de ciertos alumnos incluso de una misma aula o adaptaciones curriculares, atención a alumnos con frecuentes ingresos hospitalarios, etc.), los servicios de información a los usuarios del sistema educativo que van configurándose, etc. Los ciudadanos, en muchas ocasiones, manifiestan expectativas erróneas sobre lo que pueden encontrar en los centros educativos y entre sus profesionales. Han de saber con seguridad, por ejemplo, que de ninguna manera son guarderías laborales, que los orientadores no son psicólogos de familia, que la promoción académica depende de los criterios adoptados por los claustros y no de los deseos de los padres, que los consejos escolares o departamentales no son parlamentos sino órganos de gestión, que el aprovechamiento académico no es opcional para los alumnos de cualquier nivel, etc.
} 
Los periodistas no tienen discípulos y los profesores sí; aunque ambos profesionales aspiran a ampliar el horizonte de los conocimientos de sus contemporáneos y a hilvanarlos para mostrar que es posible y necesario que cada cual construya su sistema de convicciones como habitante que es de un mundo cada vez más complejo y más acelerado. Hay que detener la creciente sensación de que estamos sumidos en un caos cada vez mayor, del que ciertas fuerzas desconocidas y poderosísimas tienen la clave de su interpretación y gestión. Es -me parece- una de las mejores aportaciones de periodistas y profesores, sea cual sea el nivel de la audiencia o de los alumnos.

Queda una última precisión sumamente importante para delimitar el objeto de estudio del Periodismo Educativo y el de lo que Francis Desart, en los años setenta, llamó Educomunicación o educación en materia de comunicación.

El objetivo de esta disciplina es convertir en materia instrumental (como las asignaturas de lengua o de matemáticas), y no en mero auxiliar didáctico, la investigación en el aula de las características comunicativas, técnicas, empresariales y éticas de los medios de comunicación así como de las posibilidades constructivas de sus receptores ${ }^{11}$. «En el fondo se trata de intentar superar la vieja dualidad de cultura humanística y de cultura tecnológica» 12 , introduciendo nuevos códigos en los que confluyen ambas (alfabetización mediática). Los medios de comunicación y sus profesionales pueden contribuir con sus medios y su experiencia al desarrollo de esta propuesta verdaderamente innovadora, promoviendo la formación de profesores, editando cuadernos especiales periódicos, programando tiempos de radio y de televisión y sitios en Internet. Pero lo que en ellos contenga información de actualidad y sus complementos es propio del Periodismo Educativo y no de la Educomunicación o de los docentes.

La preocupación profesional de éstos, especialmente en los niveles no universitarios, es considerable, como lo demuestra la amplitud de la bibliografía puesta a su disposición y que debiera sugerir a los investigadores de la comunicación mediática un esfuerzo similar de desarrollo de una reflexión sobre la educación como objeto de información periodística y las funciones educadoras de la actividad periodística (Cfr. Bibliografía).

${ }^{11}$ UNESCO, La educación en materia de comunicación, PUF, París, 1984.

12 SÁNCHEZ BRAVO, A., «Educomunicación», en BENITO, A. (dir.), Diccionario de Ciencias y Técnicas de la Comunicación, Paulinas, Madrid, 1991. 
Por último, los conocimientos de los que ha de disponer un periodista para afrontar con éxito los objetivos señalados son, en sus grandes líneas, los siguientes:

$1^{\circ}$. Revisión de los funciones del Periodismo a la luz de los de la Educación para Toda la Vida.

$2^{\circ}$.- Nociones de Historia de la Educación: ideales pedagógicos y prácticas docentes, discentes e institucionales.

$3^{\text {o. }}$ - Protagonistas de la educación contemporánea: profesionales (docentes o no), alumnos, padres y sociedad civil.

$4^{\text {o }}$.- Fuentes del Periodismo Educativo (entre las documentales, las de Programación, especialmente).

$5^{\circ}$.- Nuevos canales para la información educacional: agencias de noticias especializadas, Internet, radio y televisión temáticas.

$6^{\circ}$.- Dimensiones éticas y políticas de la información educacional. El periodista ante una democracia que pretende garantizar un estado del bienestar en el que se incluye la educación inicial y continua. Concepto de calidad en la educación.

$7^{\circ}$.- Construcción de la agenda mediática sobre educación. Introducción a métodos de observación de este temario.

$8^{\circ}$.- La Educomunicación: alfabetización mediática y usos didácticos de los medios. Misión de los periodistas en las instituciones educativas: observación y asesoramiento (incluida la formación del personal alfabetizador). 


\section{Bibliografía (1990-1998)}

\section{A. BASES PARA EL PERIODISMO EDUCATIVO}

- AGUILERA, J.M., Aprendiendo en la era de la información, Cefocop, La Coruña, 1996.

- ALI, I., GANUZA, J.L., Internet en la educación, Anaya, Madrid, 1997.

- ÁlVAREZ TOSTADO, C., La calidad de la educación. Entre el eslogan y la utopía, Magisterio del Río de la Plata, Buenos Aires, 1997.

- APPLE, M.W., BEANE, J.A. (comps.), Escuelas democráticas, Morata, Madrid, 1997.

- BEETHAM, D., BOYLE, K., Cuestiones sobre la democracia. Conceptos, elementos y principios básicos, Los Libros de la Catarata, 1996.

- BORONAT MUNDINA, J., Medios de comunicación y educación: análisis de contenido de la prensa educativa, UNED, Palencia, 1995.

- CERVILla, E., Postmodernidad y educación, Dykinson, Madrid, 1993.

- COLOM, A.J., Teorías e instituciones contemporáneas de la educación, Ariel, Barcelona, 1997.

- CORZO, J.L., Educar(nos) en tiempos de crisis, CCS, Madrid, 1995.

- DIEZHANDINO, M.P., Periodismo de servicio. La utilidad como complemento informativo en Time, Newsweck y US News and World Report y unos apuntes del caso español, Bosch, Barcelona, 1994.

- ESTEVE, J.M., FRANCO, S., VERA, J., Los profesores ante el cambio social: repercusiones sobre la evolución de la salud de los profesores, Anthropos, Barcelona, 1995.

- FERNÁNDEZ ENGUITA, M., La escuela a examen. Un análisis sociológico para educadores y otras personas interesadas, Pirámide, Madrid, 1995.

- FERNÁNDEZ PÉREZ, M., Las tareas de la profesión de enseñar, Siglo XXI, Madrid, 1994.

- GASCÓN BAQUERO, M.C., La radio en la educación no formal, CEAC, Madrid, 1991.

- GIL, F., Sociología del profesorado, Ariel, Barcelona, 1996.

- HALliDAY, J., Educación, gerencialismo y mercado, Morata, Madrid, 1995.

- LÁZARO LORENTE, L.M., Prensa racionalista y educación en España (1901-1932), Universidad de Valencia, 1995. 
- LOSCERTALES ABRIL, F, La otra forma de ser profesor, Universidad de Sevilla, 1987; La interacción social en la educación, Reguera, Sevilla, 1992; Las escuelas de padres y los problemas sociales de la educación, Grupo Comunicación y Rol Docente, Sevilla, 1994.

- LOZANO, C., La educación en los siglos XIX y XX, Síntesis, Madrid, 1994.

- LOZANO, J.M., ¿De qué hablamos cuando hablamos de los jóvenes?, Cristianisme i Justicia, Barcelona, 1991.

- LUQUE DOMÍNGUEZ, P.A., Espacios educativos. Sobre la participación y transformación social, EUB, Barcelona, 1995.

- MARÍN IBÁÑEZ, R., Principios de la educación contemporánea, Rialp, Madrid, 1990.

- MORALES VALLEJO, P., La relación profesor-alumno en el aula, PPC, Madrid, 1998.

- MORENO M., Establecimientos escolares en transformación. El centro educativo comunitario y su rol compensatorio, Cincel, Madrid, 1993.

- OROZCO, G., Televisión y audiencias, E. de la Torre, Madrid, 1996.

- ORTEGA, F., VELASCO, A., La profesión de maestro, CIDE, Madrid, 1991.

- PEIRO, J.M., El estrés de enseñar, Alfar, Sevilla, 1991.

- PÉREZ JIMÉNEZ, J.C., Imago mundi. La cultura audiovisual, Fundesco, Madrid, 1996.

- RAIGÓN PÉREZ DE LA CONCHA, G., Periodismo y reforma educativa, Alfar, Sevilla, 1997; Una escuela tan grande como el mundo. Los periodistas y la educación de los ciudadanos, Mad, Sevilla, 1998 (en prensa).

- RAMONET, I., Un mundo sin rumbo. Crisis de fin de siglo, Debate, Madrid, 1997.

- ROSZAK, T., El culto a la información. El folclore de los ordenadores y el verdadero arte de pensar, Grijalbo, Méjico D.F., 1990.

- SAN MARTÍN ALONSO, A., La escuela de las tecnologías, Universidad de Valencia, 1995.

- SÁNCHEZ CAÑADAS, A., Aspectos Educativos en la Perspectiva de la Unidad Europea, Diputación de Almería, 1997.

- SÁNCHEZ MARTÍN, M.F., Guía para el conocimiento sociológico del medio escolar, CCS, Madrid, 1996.

- SAVATER, F., El valor de educar, Ariel, Barcelona, 1997.

- TEDESCO, J.C., Un nuevo pacto educativo (Educación, competitividad y ciudadanía en la sociedad moderna), Anaya, Madrid, 1996. 
- TREJO DELARBE, R., La nueva alfombra mágica. Usos y mitos de Internet, la red de redes, Fundesco, Madrid, 1996.

- TRILLA, J., La educación fuera de la escuela. Ámbitos no formales y educación social, Ariel, Barcelona, 1993.

- UNESCO, La educación encierra un tesoro, Santillana, Madrid, 1998.

- WANJIRU GUICHURRE, G., La ética de la profesión docente. Estudio introductorio a la deontología de la educación, Eunsa, Pamplona, 1995.

\section{B. BASES PARA LA PRÁCTICA EDUCOMUNICATIVA}

- ACUÑA LIMÓN, A. (coord.), Nuevos medios, viejos aprendizajes. Las nuevas tecnologías de la educación, Universidad Iberoamericana, México, 1995.

- AGUADED GÓMEZ, J.I., Enseñar y aprender con prensa, radio y televisión, Grupo Comunicar, Huelva, 1992; Comunicación audiovisual en una escuela renovada: propuestas desde los medios, Grupo Comunicar, Huelva, 1993; Comunicación audiovisual en una enseñanza renovada, Grupo Comunicar, Huelva, 1996.

- AGUADED GÓMEZ, J.I., CABERO, J., Educación y Medios de Comunicación en el contexto iberoamericano, Universidad de la Rábida, Huelva, 1995.

- AGUADED GÓMEZ, J.I., MORÓN MARCHENA, J.A. (coords.), Enseñar y aprender la actualidad con los medios de comunicación, Grupo Comunicar, Huelva, 1994.

- AGUILAR, P., Manual del espectador inteligente, Fundamentos, Madrid, 1996.

- AlOnso, M., MATIlla, L., VÁZQUEZ, M., Teleniños públicos, teleniños privados, La Torre, Madrid, 1995.

- ALONSO, M., MATILLA, L., Imágenes en acción, Análisis y práctica de la expresión audiovisual en la escuela activa, Akal, Madrid, 1990.

- APARICI, R., La revolución de los medios audiovisuales. Educación y nuevas tecnologías, E. de la Torre, Madrid, 1996.

- AYUSTE, A., FLECHA, R., LÓPEZ PALMA, F., LLERAS, J., Planteamientos de la pedagogía crítica, Comunicar y transformar, Grao, Barcelona, 1994.

- BALLESTA PAGÁN, F.J., La incorporación de la prensa a la escuela, Seco Olea, Madrid, 1991; Medios de comunicación y nuevas tecnologías, Diego Marín, Murcia, 1997. 
- BALlESTA PAGÁN, F.J (Coord.), Enseñar con los medios de comunicación, PPU/DM, Barcelona, 1995.

- BARTOLOMÉ CRESPO, D., Prensa en las aulas, Edinford, Málaga, 1993; Prensa en las aulas 2, 1996.

- BARTOLOMÉ CRESPO, D., SEVILLANO GARCÍA, M.L., Enseñanzaaprendizaje con los medios de comunicación en la reforma, Madrid, 1991; Enseñanza-aprendizaje con medios de comunicación y nuevas tecnologías, UNED, Madrid, 1997.

- BERGONDO LlORENTE, E., Comunicación Audiovisual. Materiales didácticos del MEC. Optativas de Bachillerato, MEC, Madrid, 1993.

- BIEDMA LÓPEZ, J., Imágenes e ideas, Gráficas Úbeda, Jaén, 1997.

- BLÁZQUEZ ENTONADO, J., CABERO ALMENARA, J., LOSCERTALES ABRIL, F. (coords), En memoria de José Manuel López-Arenas. Nuevas tecnologías de la información y de la comunicación para la educación, Alfar, Sevilla, 1994.

- BRAVO, J.L., Criterios para la evaluación de videogramas educativos, ICE Universidad Politécnica, Madrid, 1992.

- BROWN, L.K, Cómo utilizar bien los medios de comunicación. Manual para padres y maestros, Visor, Madrid, 1991.

- CABERO AlMENARA, J., Primeras Jornadas sobre Medios de Comunicación, Recursos y Materiales para la Mejora Educativa, Secretariado de Recursos Audiovisuales y Nuevas Tecnologías, Sevilla, 1994.

- CABERO ALMENARA, J., CERDEIRA GAYOL, M, GÓMEZ FERNÁNDEZ, G., Medios de comunicación, recursos audiovisuales y materiales para la mejora educativa 2, Secretariado de Recursos Audiovisuales y Nuevas Tecnologías, Sevilla, 1996.

- CAMPUZANO RUIZ, A., Tecnologías audiovisuales y educación. Una visión desde la práctica, Akal, Madrid, 1992.

- COLECTIVO ONDA ESCOLAR, La radio escolar: experiencia y aplicaciones didácticas, CECJA Delegación Provincial de Cádiz, 1993.

- CORREDERA APARICIO, M., Experiencias escolares en torno a la radio, Centro de Profesores de Salamanca, 1994.

- CORREA GARCÍA, R.I., La imagen que se esconde, AIQB, Huelva, 1994.

- CHARLES CREEL, M., OROZCO GÓMEZ, G. (coords.), Educación para la recepción. Hacia una lectura crítica de los medios, Trillas, México D.F., 1990.

- ECHEVERRÍA, L., Cosmopolitas domésticos, Anagrama, Barcelona, 1995. 
- FANDOS IGADO, M., Juega con la imagen. Imagina juegos. Hacia una integración curricular de los medios de comunicación en la enseñanza, Grupo Comunicar, Huelva, 1995.

- FERIA MORENO, A., Unidades didácticas de prensa en Educación Primaria, Grupo Comunicar, Huelva, 1993.

- FERIA MORENO, A. (dir.), Educación y televisión, Grupo Comunicar, Huelva, 1995.

- FERIA MORENO, A., MORÓN MARCHENA, A. (dirs.), Educación y publicidad, Grupo Comunicar, Huelva, 1997.

- FERNÁNDEZ MARTÍNEZ, T., El universo de papel: trabajamos con el periódico en el aula, Grupo Comunicar, Huelva, 1997.

- FERRES I PRATS, J., Vídeo y educación, Paidós, Barcelona, 1992; La publicidad, modelo para la enseñanza, Akal, Barcelona, 1994; Televisión y educación, Paidós, Barcelona, 1997.

- FERRÉS Y PRATS, J., MARQUÉS GRAELLS, P. (coords.), Comunicación educativa y nuevas tecnologías, Praxis, Barcelona, 1996.

- FREIRE, P., Niños, medios de comunicación y su conocimiento, Herder, Barcelona, 1992.

- GALLEGO ARRUFAT, M.J. (dir.), El profesorado y la televisión, Universidad, Granada, 1997.

- GALlEGO GIL, D.J., ALONSO GARCÍA, C.M., CANTÓN, I. (coords), Integración curricular de los recursos tecnológicos, Oikos-Tau, Barcelona, 1996.

- GARCÍA HOZ (dir.), La personalización educativa en la sociedad informatizada, Rialp, Madrid, 1995.

- GARCÍA NOVELL, F., Inventar el periódico. Propuestas para trabajar la prensa, La Torre, Madrid, 1992.

- GRUPO IMAGO, Imagotopías. Aproximaciones al estudio de las imágenes, Granada, 1993; El discurso de la televisión. Teoría y Didáctica del medio televisivo, Granada, 1994.

- GRUPO SPECTUS, Aprende conmigo. La televisión en el centro educativo, MEC/La Torre, Madrid, 1996.

- GUTIÉRREZ MARTÍN, A., Educación multimedia y nuevas tecnologías, E. de la Torre, Madrid, 1997.

- JIMÉNEZ, A. (ed.), Comunicación y educación, Colegio de Doctores, Granada, 1996. 
- LOMAS, C., El espectáculo del deseo. Usos y formas de la persuasión publicitaria, Octaedro, Barcelona, 1996.

- LÓPEZ CUBINO, R., La prensa en la escuela: orientaciones didácticas para su utilización, Escuela Española, Madrid, 1997.

- LUNA ARCOS, F., ARRI MARTÍNEZ, A., EGAÑA GIMÉNEZ, A., Taller de prensa. Prensa tailerra, Dirección de Renovación Pedagógica, Álava, 1997.

- MARCH CERDA, J.C., La generación de la televisión, Escuela Andaluza de Salud Pública, Granada, 1994.

- MARTÍN CATALÁN, D., Las tecnologías de la información en la educación. España, PNTIC.MEC, Madrid, 1991.

- MARTÍNEZ SÁNCHEZ, E., El periódico en la educación de las personas adultas, Grupo Comunicar, Huelva, 1994.

- MASTERMAN, L., La enseñanza de los medios de comunicación, E. de la Torre, Madrid, 1993.

- MEC, La televisión educativa en España. Informe Marco, MEC, Madrid, 1996.

- MESO AYERDI, K., Guía de navegación. Educación en Internet, Anaya, Madrid, 1997.

- MOLINA, L., El vídeo: uso pedagógico y profesional en la escuela, Alta Fulla, Barcelona, 1990.

- MORDUCHOWICZ, R., La escuela y los medios, Aique, Buenos Aires, 1997.

- MORENO HERRERO, I., La radio en el aula: posibilidades para comunicar de forma creativa (material curricular de Lengua y Literatura Española y Comunicación, ESO, $2^{\circ}$ ciclo), Octaedro, Barcelona, 1997.

- MUÑOZ GONZÁlEZ, J.J., Radio educativa, Librería Cervantes, Salamanca, 1994.

- MUÑOZ, J.J., PEDRERO, L.M., La televisión y los niños, Librería Cervantes, Salamanca, 1996.

- ORTEGA NÚÑ̃Z, Esther, Televisión educativa-Instructional television, edición de la autora (San Pablo, 12-A-4C, 09002-Burgos), 1994. 12 págs.

- PEÑUELAS AYLLÓN, M.A., LÓPEZ LÓPEZ, J.F., La radio como medio versátil en educación, edición de Miguel Ángel Peñuelas (Residencial Tarpeya, 55, 45007-Toledo), 1995. 50 págs.

- PEREZ TORNERO, J.M., El desafío educativo de la TV, Paidós, Barcelona, 1994; Televisión educativa, UNED, Madrid, 1993. 
- PABLOS PONS, J. (coord.), La Tecnología Educativa en España. Actas de las Primeras Jornadas Universitarias de Tecnología Educativa, Universidad de Sevilla, 1994.

- PABLOS PONS, J., GORTARI DRETS, C., Las nuevas tecnologías de la información en la educación, Alfar, Sevilla, 1992.

- PEÑA, R., La educación en Internet. Guía para su aplicación práctica en la enseñanza, Inforbook's, Barcelona, 1997.

- PRADAS LÓPEZ, A., La escuela en el dial de «Radio Ribera», Diputación Provincial, Sevilla, 1996.

- RADIO ECCA, Memoria de la fundación ECCA: 1989-90, Radio Ecca, Las Palmas de Gran Canaria, 1991.

- RICO OLIVER, L., El buen telespectador. Cómo ver y enseñar a ver la televisión, Espasa Calpe, Madrid, 1994.

- RÍO APARICIO, P., La radio en el diseño curricular, Bruño, Madrid, 1990.

- RODRÍGUEZ DIÉGUEZ, J.L., SÁENZ BARRIO, O. (coords.), Tecnología educativa. Nuevas tecnologías aplicadas a la educación, Marfil, Alcoy, 1995.

- ROMERO LÓPEZ, a. (Coord.), Lenguajes y enseñanzas, Fundación Educación y Futuro, Granada, 1996.

- RUIZ LÓPEZ, M.C., MEDINA BARRAGÁN, F.J., RUIZ LÓPEZ, D., Estudiamos con la prensa: experiencias, Centro de Estudios Bit a Bit, Jaén, 1996.

- SÁNCHEZ, J., DÍAZ, C., Alfabetización visual, La Torre, Madrid, 1995.

- SEVILlANO GARCÍA, M.L., BARTOLOME CRESPO, D., Enseñar y aprender con la prensa: fichas y modelos de trabajo, CCS, Madrid, 1995.

- TEJEDOR, F.J., VALCÁRCEL, A., Perspectivas de las Nuevas Tecnologías en la Educación, Narcea, Madrid, 1996.

- VV.AA., Alfabetización y postalfabetización a través de la radio, Popular, Madrid, 1992.

- VV.AA., La otra mirada a la tele. Pistas para un consumo inteligente de la televisión, Junta de Andalucía, Sevilla, 1997.

- VILlAR ANGUlO, L.M., CABERO ALMENARA, J. (dirs.), Desarrollo profesional docente en nuevas tecnologías de la información y de la comunicación, Grupo de Investigación Didáctica, Sevilla, 1997.

- YOUNIS HERNÁNDEZ, J.A., El aula fuera del aula: educación invisible, comunicación audiovisual, Nogal, Las Palmas de Gran Canaria, 1993. 